Research paper

\title{
The relationship between cognitive distortion, depressive symptoms, and social adaptation: A survey in Japan
}

\author{
Maki Ota $^{\mathrm{a}, \mathrm{b}, *}$, Shinya Takeda ${ }^{\mathrm{c}}$, Shenghong Pu ${ }^{\mathrm{d}}$, Hiroshi Matsumura ${ }^{\mathrm{e}}$, Takayuki Araki ${ }^{\mathrm{d}}$, \\ Naoko Hosoda ${ }^{\mathrm{f}}$, Yoko Yamamoto ${ }^{\mathrm{g}}$, Aya Sakakihara ${ }^{\mathrm{h}}$, Koichi Kaneko ${ }^{\mathrm{d}}$ \\ ${ }^{a}$ Division of Neuropsychiatry, Department of Clinical Medicine, Tottori University Graduate School of Medical Sciences, Yonago, Tottori 683-8503, Japan \\ ${ }^{\mathrm{b}}$ National Hospital Organization, Tottori Medical Center, Tottori-shi, Tottori 689-0203, Japan \\ ${ }^{\mathrm{c}}$ Department of Clinical Psychology, Tottori University Graduate School of Medical Sciences, Yonago, Tottori 683-8503, Japan \\ ${ }^{\mathrm{d}}$ Division of Neuropsychiatry, Department of Brain and Neuroscience, Tottori University Faculty of Medicine, Yonago, Tottori 683-8503, Japan \\ ${ }^{\mathrm{e}}$ Kurayoshi Hospital, Kurayoshi, Tottori 682-0023, Japan \\ ${ }^{\mathrm{f}}$ Division of Neuropsychiatry, Yowa Hospital, Yonago, Tottori 683-0841, Japan \\ ${ }^{\mathrm{g}}$ Department of Fundamental Nursing, School of Health Science, Tottori University Faculty of Medicine, Yonago, Tottori 683-8503, Japan \\ ${ }^{\text {h }}$ Community Health Nursing, Faculty of Medicine, Shimane University, Izumo, Shimane 693-8501, Japan
}

\section{A R T I C L E I N F O}

\section{Keywords:}

Cognitive distortion

Social adaptation

Depressive symptom

\begin{abstract}
A B S T R A C T
Background: In the treatment of depression, improvements in both clinical symptoms and social adaptation are important. Previous studies have shown that cognitive distortion and depressive symptoms are mutually related, and that depressive symptoms and social adaptation are related to each other. However, it is unknown how these three factors interrelate. Therefore, this study examined the relationship between cognitive distortion, depressive symptoms, and social adaptation.

Methods: The final analyzed sample consisted of 430 employees of a manufacturing company in Japan (74.2\% male, $24.7 \%$ female, $1.2 \%$ unknown). Participants completed the Worker's Cognitive Distortion Scale (WCDS), Beck Depression Inventory-Second Edition (BDI-II), and Social Adaptation Self-Evaluation Scale (SASS). The WCDS was further divided into two subscales: self-contained cognitive distortion (WCDS-S) and environmentdependent cognitive distortion (WCDS-E). We used a covariance structure analysis for the main analysis and examined the relationship between these three variables' scores.

Results: The results revealed that both the WCDS-S and WCDS-E affected social adaptation indirectly via depressive symptoms, and that the WCDS-S additionally affected social adaptation directly. It was further revealed that the WCDS-S exerted a greater effect on depressive symptoms than the WCDS-E.

Limitations: The participants were healthy cases. As such, one must be cautious about applying the results of healthy cases to clinical cases.

Conclusions: This study indicates that cognitive distortion affects social adaptation directly and that it is indirectly mediated by depressive symptoms. Thus, professionals are required to attempt to treat depressive symptoms and improve social adaptation by considering that interventions in cognitive distortion may be effective.
\end{abstract}

\section{Introduction}

Over the years, efforts to treat depression have focused on improving depressive symptoms. However, there is now increasing attention on improving social adaptation (Kupferberg et al., 2016; Sheehan et al., 2017). Social adaptation has been particularly relevant to industry, where many employers face an increasing number of leaves of absence due to psychiatric conditions (Organisation for Economic
Co-operation and Development, 2012), high depression relapse rates (Angst, 1997), and problems in reintegrating employees back into work (Ervasti et al., 2013). In view of these problems, researchers believe that a person's readiness to return to work should be determined not only by an improvement in depressive symptoms but also by a satisfactory level of social adaptation.

According to Bosc (2000), social adaptation concerns the interactions between an individual and the environment, and it describes the

\footnotetext{
* Corresponding author.

E-mail address: oota.maki.yh@mail.hosp.go.jp (M. Ota).
} 
individual's ability to perform professional and social roles, including with respect to one's partner or family. Accordingly, Bosc et al. (1997) developed the Social Adaptation Self-Evaluation Scale (SASS) premised on the behavioral model. In the behavioral model (Libet and Lewinsohn, 1973; Coyne, 1976; Rehm, 1977; Lewinsohn et al., 1979; Seligman, 1981), it is considered that exposure to stress leads to depressive symptoms, and depressive symptoms reduce opportunities for adaptive behavior and thus decrease opportunities for positive reinforcement, resulting in impaired social adaptation. Simply put, altered social adaptation is a secondary effect that results from depressive symptoms.

Some studies have examined the role of pharmacotherapy in approaches for improving social adaptation and suggested that some antidepressants are effective in improving social adaptation (Healy, 2000; Keller, 2001; Briley and Moret, 2010). The behavioral model of depression suggests a mechanism by which pharmacotherapy improves social adaptation; that is, as the pharmacotherapy reduces the depressive symptoms, the patient feels more motivated to engage in adaptive behavior, which creates more opportunities for positive reinforcement.

Although drugs can improve symptoms, the next steps in the recovery process may depend on how empowered the individual becomes and how well they can access environmental resources.

Hence, when examining the effect of an intervention for improving social adaptation, one must consider the role of several variables involved in the process, in addition to the symptoms.

According to Beck's cognitive theory, one possible variable is cognition (Beck, 1976, 1979). Individuals with depression may have underlying cognitive patterns that increase their risk of relapse after their depressive symptoms improve (Hedlund and Rude, 1995). In other words, their depression schema may easily reactivate as they gain more opportunities for social engagement. Thus, cognitive therapy (CT) based on Beck's cognitive theory may help to improve social adaptation because it addresses the distorted cognition that can increase the risk of relapse. There is plenty of convincing evidence that CT is effective for treating depression and preventing relapse (Matsunaga et al., 2010; Marco et al., 2018). Nonetheless, no studies have examined the relationship between cognitive variables, depressive symptoms, and social adaptation.

However, the literature does offer some clues about this relationship. The SASS, which is a measure of social adaptation in individuals with depression, posits that depressive symptoms predict social adaptation (Bosc, 2000), suggesting a causal pathway from depressive symptoms to social adaptation. As for the relationship between depressive symptoms and cognitive variables, the cognitive model posits that cognition predicts emotion and behavior (Beck, 1976). There is also a causal pathway from the cognitive variables to depressive symptoms. According to the cognitive theory, emotion generates behavior (Weishaar, 1993). Thus, we can assume a causal chain between the three variables, whereby cognition leads to depressive symptoms, and depressive symptoms lead to social adaptation.

The particular cognitive variable that we focused on in the present study was cognitive distortion.

In this study, cognitive distortion was measured using the Worker's Cognitive Distortion Scale (WCDS; Ota et al., 2014). WCDS is useful for measuring cognitive distortions that be easy to occur in the workplace, and that cause biased recognition on things happening in the environment.

Its 15 items are divided into two subscales: self-contained cognitive distortion (WCDS-S) and environment-dependent cognitive distortion (WCDS-E). The following is an example of an item of the WCDS-S subscale: "If I complete a task but am not satisfied with the result, I believe that I have failed the task." A high score for this factor indicates the presence of cognitive distortion that has resulted from judging matters using one's own criteria, without being influenced by others. The following is an example of an item of the WCDS-E subscale: "When others seem upset, I assume that I must have done something to upset them." A high score on this factor indicates that there is cognitive distortion that has resulted from dependency on the social environment and negative thinking. The WCDS is reported to have excellent reliability and validity (Ota et al., 2014). It is useful in the following ways: it was developed for use with workers and it has a two-factor structure. Therefore, it is possible to examine cognitive distortion in more detail.

From the above, we assumed a causal pathway in the order of cognitive distortion, depressive symptoms, and social adaptation, and we examined the pathway using covariance structure analysis. This originality of this research is that it examined these relationships. Further, this study used an analog design and assumed that depression is on a continuum with a healthy state (e.g., Flett et al., 1997; Cox et al., 1999).

\section{Methods}

This is a cross-sectional study using a questionnaire.

\subsection{Participants}

The participants were 550 employees of manufacturing company in Shimane, Japan.

We requested this company's cooperation for this study based on two points: it is a large-scale company with more than 300 employees and it has putting efforts into ensuring the mental health of its employees. Through the company's office, instructions and the questionnaire were distributed to each employee. They received an overview of the study and a written explanation, and participants who gave their informed consent completed the questionnaire. A response was obtained from 490 people, and the analysis target was reached as 430 people provided valid responses (effective response rate: $87.8 \% ; 74.2 \%$ male, $24.7 \%$ female, $1.2 \%$ unknown). The sample size was calculated to have a confidence coefficient of $95 \%$, a confidence interval of $10 \%$ or less, and a Japanese worker rate (population rate) of about $60 \%$, resulting in a required sample size of 369 . The number of participants in this study meets this criterion. The explanation provided to participants stated the following: the data gathered in this study would be analyzed so that individuals could not be identified, only the data of those who consented would be analyzed, and no disadvantages would arise because of consenting or not consenting to participate in the study. This study was approved by the Institutional Review Board of the Faculty of Medicine, Tottori University (authorization number 1703A228).

\subsection{Measures}

The questionnaire collected data about the participants' personal attributes and included the three scales to measure cognitive distortion, depressive symptoms, and social adaptation.

\subsubsection{Personal attributes}

The questionnaires included the following items describing the participants' personal attributes: age, sex, occupation, form of employment, consultation history of psychiatric or psychosomatic disorders (yes/no), family history of illness (yes/no), chronic physical illness (yes/no), living with the family (yes/no), number of years in the present job (years of experience), and level of social support.

The level of social support was assessed with the following three questions, each of which was rated on a 4-point scale (ranging from 
completely disagree to completely agree; $0-3$ ): "Do you have someone in your life whom you could rely on for support in times of trouble?" "Do you have someone to talk to when you are sick in bed?" and "Do you have someone in your life with whom you can discuss your worries and problems?" The level of social support was thus scored from 0 to 9 , with a higher score indicating a higher level of social support.

\subsubsection{Depressive symptoms}

Depressive symptoms were measured using the Beck Depression Inventory-Second Edition (BDI-II) (Beck et al., 1996). The BDI-II is a 21item self-report scale for measuring the severity of depressive symptoms. Each of the 21 items corresponds to a symptom of depression and is scored on a 4-point scale (consisting of four statements); the score indicates the severity of the symptom in question. The score for each item ranges from 0 to 3 , and the total score ranges from 0 to 63 . The Japanese version of the scale was used in this study, which was developed and validated by Kojima et al. (2002). According to Beck et al. (1996), the cut-off score for depression is 17 (a score of 17 or more indicates that depression is present). In the present study, the BDIII had an internal consistency (coefficient $\alpha$ ) of 0.92 , and the original standard is 0.87 .

\subsubsection{Cognitive distortion}

Cognitive distortion was measured using Ota et al. (2014) WCDS (supplementary material). The WCDS is a 15-item self-report scale for measuring cognitive distortion in an occupational context. Factor analysis has revealed that the 15 items are divided into two subscales: the WCDS-S (9 items) and WCDS-E (6 items). Each item is rated on a 4point scale ( $0=$ never, $1=$ hardly ever, $2=$ usually, and $3=$ always).

The WCDS is reported to have excellent reliability and validity (Ota et al., 2014). With regard to reliability, internal consistency of WCDS was evaluated with Cronbach's alpha, and findings indicated excellent internal consistency (WCDS-S $=0.91$, WCDS-E $=0.81$ ). Concurrent validity was evaluated by calculating Pearson's product moment correlation coefficient between the WCDS total score and each factor score, and the 12-item General Health Questionnaire (GHQ-12) total score and state anxiety of State-Trait Anxiety Inventory (STAI-S). Findings indicated that the GHQ-12 was moderately correlated with the WCDS total score $(r=0.58, p<.001)$, WCDS-S $(r=0.56, p<.001)$, and WCDS-E $(r=0.50, p<.001)$, and that the STAI-S was moderately correlated with the WCDS total score $(r=0.54, p<.001)$, WCDS-S $(r=0.52, p<.001)$ and WCDS-E $(r=0.48, p<.001)$. Construct validity was evaluated by Freiss's kappa coefficient. Regarding the WCDS 15 items, the rate of agreement between each item and cognitive distortion was evaluated by 5 evaluators (graduate students majoring in clinical psychology). As a result, the kappa coefficient was $0.94(95 \%$ $\mathrm{CI}=0.88-1.00$ ), and a high rate of agreement between raters was obtained.

The following is an example of an item of the WCDS-S subscale: "If I complete a task but am not satisfied with the result, I believe that I have failed the task." A high score for this factor indicates cognitive distortion resulting from judging matters using one's own criteria, without being influenced by others. The following is an example of an item of the WCDS-E subscale: "When others seem upset, I assume that I must have done something to upset them." A high score for this factor indicates cognitive distortion resulting from dependency on the social environment and negative thinking. The total score for the WCDS ranges from 0 to 45 , with a higher score indicating stronger cognitive distortion. In the present study, the WCDS, WCDS-S, and WCDS-E had internal consistencies (coefficient $\alpha$ ) of 0.92, 0.91, 0.79, respectively.

\subsubsection{Social adaptation}

Social adaptation was measured using Bosc et al. (1997) SASS. The
SASS is a 21-item self-report scale for measuring the social functioning of individuals with depression. The scale covers four broad areas of social functioning: work, spare time, family, and organizing and coping with the environment. Participants answer questions about their motivations, self-awareness, and satisfaction regarding the different roles they play in their daily lives, including their roles as parents, partners, colleagues, and friends. The score for each item ranges from 0 to 3 , and the total score ranges from 0 to 60 . The Japanese version of the scale was used in this study, which was developed and validated by Goto et al. (2005). A score of 35-52 indicates a normal level of social functioning ( $80 \%$ of the population falls within this range). A score of less than 25 indicates impaired social functioning (Ueda et al., 2011). In the present study, the SASS had an internal consistency (coefficient $\alpha$ ) of 0.81 , and the original standard is 0.76 .

\subsection{Statistical analyses}

All of the statistical analyses were performed using the SPSS Ver.25 (IBM Corp., Armonk, NY, USA) and AMOS 24.0 software (IBM Corp., Armonk, NY, USA). First, we examined the personal attributes and the average (mean \pm standard deviation) scores on each scale. Next, we examined two models of the relationship between cognitive distortion, depressive symptoms, and social adaptation; one examined their relationship with the WCDS and the other examined their relationship with the two subordinate scales of the WCDS.

In the first model review, the Pearson correlation coefficients and partial coefficients between the three scales (the total scores of the WCDS, BDI-II, and SASS) were calculated. Next, a covariance structure analysis was performed to examine the model. The WCDS, BDI-II, and SASS were entered into this model. In this analysis, we assumed that there were two pathways: an indirect pathway from the WCDS to the SASS with the BDI-IIas the mediator, and a direct pathway from the WCDS to the SASS.

As mentioned previously, the WCDS consists of two subscales (the WCDS-S and WCDS-E). As such, we assumed that each subscale could separately correlate with depressive symptoms and social adaptation. In this analysis, we also assumed two pathways: an indirect pathway from the two WCDS subscales to the SASS with the BDI-II as the mediator, and a direct pathway from the WCDS subscale to the SASS. Accordingly, the Pearson correlation coefficients and partial coefficients between each subscale of the WCDS (i.e., the WCDS-S and WCDS-E), and the BDI-II and SASS were calculated. Then, the variables were modeled, and the data were subjected to a covariance structure analysis. In the covariance structure analyses, the pathways exhibiting nonsignificant partial coefficients were removed from the saturated model until an optimum model was identified.

The measures used to test the model were the chi-square test statistic $\left(\chi^{2}\right)$, goodness-of-fit index (GFI), adjusted goodness-of-fit index (AGFI), comparative fit index (CFI), and the root mean square error of approximation (RMSEA); the confidence interval (CI) was $90 \%$ in each case. The following results were deemed to indicate an acceptable fit: GFI $>0.90$, AGFI $>0.85$, CFI $>0.95$, and RMSEA $<0.08$. The following results were deemed to indicate a good fit: GFI $>0.95$, AGFI $>0.90$, CFI $>0.97$, and RMSEA $<0.05$ (SchermellehEngel et al., 2003).

\section{Results}

\subsection{Personal attributes and average (mean \pm standard deviation) scores}

Table 1 shows the participants' personal attributes. Table 2 shows the results for each scale.

Regarding the personal attributes, $92.4 \%$ of the sample was $20-59$ 
Table 1

Attributes of subjects.

\begin{tabular}{|c|c|c|c|}
\hline Attribute & Answer & \multicolumn{2}{|c|}{$N(430)$} \\
\hline \multicolumn{4}{|l|}{ Age } \\
\hline & 10 's & 10 & 2.3 \\
\hline & 20 's & 79 & 18.4 \\
\hline & 30 's & 120 & 27.9 \\
\hline & 40 's & 97 & 22.6 \\
\hline & 50 's & 101 & 23.5 \\
\hline & 60 's & 22 & 5.1 \\
\hline & Unknown & 1 & 0.2 \\
\hline \multirow[t]{3}{*}{ Sex } & Male & 319 & 74.2 \\
\hline & Female & 106 & 24.7 \\
\hline & Unknown & 5 & 1.2 \\
\hline \multirow[t]{3}{*}{ Occupation } & Manufacturing & 417 & 97.0 \\
\hline & Services & 12 & 2.8 \\
\hline & Clerk & 1 & 0.2 \\
\hline \multirow[t]{3}{*}{ Form of employment } & Full time & 315 & 73.3 \\
\hline & Non-full time & 113 & 26.3 \\
\hline & Unknown & 2 & 0.5 \\
\hline \multirow{2}{*}{$\begin{array}{l}\text { Consultation history of psychiatric or } \\
\text { sychosomatic disorders }\end{array}$} & Yes & 46 & 10.7 \\
\hline & No & 384 & 89.3 \\
\hline \multirow[t]{2}{*}{ Family history of illness } & Yes & 81 & 18.8 \\
\hline & No & 349 & 81.2 \\
\hline \multirow[t]{2}{*}{ Chronic physical illness } & Yes & 80 & 18.6 \\
\hline & No & 350 & 81.4 \\
\hline \multirow[t]{3}{*}{ Living with family } & Yes & 318 & 74.0 \\
\hline & No & 111 & 25.8 \\
\hline & unknown & 1 & 0.2 \\
\hline $\begin{array}{l}\text { Years of experience } \\
\text { (Mean } \pm \text { SD) }\end{array}$ & & \multicolumn{2}{|c|}{$14.0 \pm 12.7$} \\
\hline $\begin{array}{l}\text { Social Support } \\
\text { (Mean } \pm \text { SD) }\end{array}$ & & \multicolumn{2}{|c|}{$6.6 \pm 2.2$} \\
\hline
\end{tabular}

SD: Standard Deviation.

Table 2

Mean and standard deviation of each rating scale.

\begin{tabular}{llll}
\hline & $N(430)$ & $\%$ & Mean (SD) \\
\hline $\begin{array}{l}\text { BDI-II } \\
<17\end{array}$ & 292 & 67.9 & $13.63(9.43)$ \\
$\geqq 17$ & 138 & 32.1 & \\
WCDS & & & $20.18(8.12)$ \\
WCDS-S & & & $11.31(5.41)$ \\
WCDS-E & & & \\
SASS & 77 & 17.9 & $31.45(7.07)$ \\
$\leqq 25$ & 212 & 49.3 & \\
$26-34$ & 141 & 32.8 & \\
$\geqq 35$ & & & \\
\hline
\end{tabular}

SD:Standard Deviation.

BDI-II: The Beck Depression Inventory-Second Edition.

WCDS:Worker's Cognitive Distortion Scale.

WCDS-S:Cognitive distortion of self-contained.

WCDS-E:Cognitive distortion of environment-dependent.

SASS: Social Adaptation Self-Evaluation Scale.

years of age. Men outnumbered women, accounting for $74.2 \%$ of the sample. As almost as $97.0 \%$ of the participants were working in manufacturing, and $73.3 \%$ were full-time. Approximately $80 \%$ of the responses about a history of seeking specialist consultations, a family history of illness, and chronic physical illness were negative. The average (mean \pm standard deviation) level of social support was $6.6 \pm 2.2$.

For the BDI-II's total scores, $138(32.1 \%)$ of the participants were suffering from depressive symptoms, as indicated by a BDI-II score of at least 17 (the cut-off for depression screening). Regarding the SASS's
Table 3

Correlations and partial correlation between total scores of WCDS, BDI-II, and SASS.

\begin{tabular}{lllc}
\hline & BDI-II & WCDS & SASS \\
\hline BDI-II & 1 & - & - \\
WCDS & $0.57^{* * * *}\left(0.47^{* * *}\right)$ & 1 & - \\
SASS & $-0.57^{* * *}\left(-0.47^{* * *}\right)$ & $-0.37^{* * *}(-0.07)$ & 1 \\
\hline
\end{tabular}

( ): partial correlation $* p<.05, * * p<.01, * * * p<.001$.

BDI-II: The Beck Depression Inventory-Second Edition.

WCDS:Worker's Cognitive Distortion Scale.

SASS: Social Adaptation Self-Evaluation Scale.

total scores, $141(32.8 \%)$ had a normal level of social functioning, as indicated by a SASS score of 35-52; $212(49.3 \%)$ were borderline, with scores ranging from 26 to 34; and 77 (17.9\%) had impaired social functioning, as indicated by a score of 25 or less. Given the lifetime prevalence of depression in Japan (Ishikawa et al., 2016), the sample featured a relatively high rate of depressive symptoms and a relatively low level of social adaptation.

\subsection{Causal relationship between the WCDS, BDI-II, and SASS}

\subsubsection{Correlation coefficients and partial coefficients}

Table 3 shows the Pearson correlations coefficients and the partial coefficients between the total scores of the WCDS, BDI-II, and SASS. The correlation coefficients calculated for each of the three pairs of the two scales were all significant. The WCDS was correlated moderately with the BDI-II $(r=0.57, p<.001)$ and with the SASS $(r=-0.37$, $p<.001$ ). According to the partial coefficients, the BDI-II was correlated moderately with the WCDS $(r=0.47, p<.001)$ and SASS $(r=-0.47, p<.001)$. On the other hand, the partial correlation coefficient between the WCDS and SASS was not significant $(r=-0.07, p=0.15)$.

\subsubsection{Covariance structure analysis}

A covariance structure analysis was performed to determine the causal relationships between the three scales. We removed pathways (from among those shown in Table 3) when the two variables did not exhibit a significant correlation coefficient, leaving a model fit for the analysis, as shown in Fig. $1\left(\chi^{2}=2.126 ; d f=1, n s ; \mathrm{GFI}=0.997\right.$; AGFI $=0.980$; CFI $=0.997$; RMSEA $\leq=0.08$; with $90 \%$ CIs: 0-0.150). The model in Fig. 1 satisfied the goodness-of-fit criteria (an acceptable fit: GFI $>0.90$, AGFI $>0.85$, CFI $>0.95$, and RMSEA $<0.08$ ), and the result indicated that the first of the two assumed pathways was correct; that is, that cognitive distortion indirectly affects social adaptation via depressive symptoms.

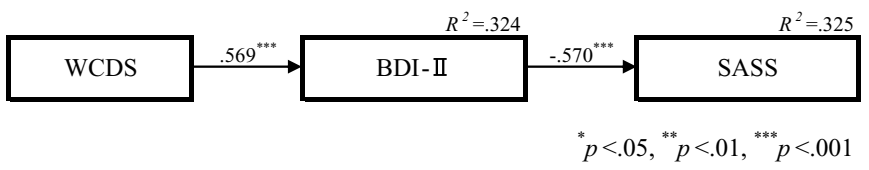

Fig. 1. The causal model between WCDS, BDI-II, and SASS. A covariance structure analysis was performed to determine the causal relationships between the three scales. We assumed that there were two pathways: an indirect pathway from WCDS to SASS with BDI-II as the mediator, and a direct pathway from WCDS to SASS. We removed pathways (from among those shown in Table 3 ) when the two variables did not exhibit a significant correlation coefficient. This model showed good fit based on fit indices $\left(\chi^{2}=2.126, d f=1\right.$, n.s.; CFI = .997; RMSEA $=.051$, with90\%Cls: 0-0.150). 
Table 4

Correlations and partial correlation between WCDS-S, WCDS-E, BDI-II, and SASS.

\begin{tabular}{lllll}
\hline & BDI-II & WCDS-S & WCDS-E & SASS \\
\hline BDI-II & 1 & - & - & - \\
WCDS-S & $0.57^{* * *}\left(0.26^{* * *}\right)$ & 1 & - & - \\
WCDS-E & $0.48^{* * *}\left(0.13^{* *}\right)$ & $.74^{* * *}\left(0.65^{* * *}\right)$ & 1 & - \\
SASS & $-0.57^{* * *}(-0.47 *)$ & $-0.39^{* * *}\left(-0.12^{*}\right)$ & $-0.28^{*}(0.07)$ & 1 \\
\hline
\end{tabular}

( ): partial correlation $* p<.05, * * p<.01$, ***p $<.001$.

BDI-II: The Beck Depression Inventory-Second Edition.

WCDS:Worker's Cognitive Distortion Scale.

SASS: Social Adaptation Self-Evaluation Scale.

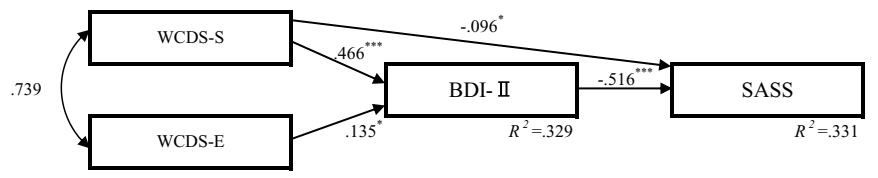

$$
{ }^{*} p<.05, * * p<.01, * * * p<.001
$$

Fig. 2. The causal model between two subscales of WCDS, BDI-II, and SASS. The WCDS consists of two subscales (the WCDS-S and WCDS-E). We assumed that each subscale of WCDS could separately correlate with depressive symptoms and social adaptation.

Therefor a covariance structure analysis was performed to determine the causal relationships between the WCDS-S, WCDS-E, BDI-II, and SASS. We also assumed two pathways: an indirect pathway from the two WCDS subscale to SASS with BDI-II as the mediator, and a direct pathway from the WCDS subscale to SASS. We removed pathways (from among those shown in Table 4) when the two variables exhibited a nonsignificant correlation coefficient. This model showed good fit based on fit indices $\left(\chi^{2}=1.797, d f=1\right.$, n.s.; CFI = .999; RMSEA $=.043$, with90\%Cls: 0-0.144).

The WCDS predicted the BDI-II $(\beta=0.569, p<.001)$, explaining $32.4 \%$ of the variance of the BDI-II. The BDI-II predicted the SASS directly $(\beta=-0.570, p<.001)$, with the WCDS and BDI-II explaining $32.5 \%$ of the variance of the SASS. The indirect effect of the WCDS on this outcome was $\beta=-0.325$.

\subsection{Causal relationships between the WCDS subscales, the BDI-II, and the SASS}

\subsubsection{Correlation coefficients and partial coefficients}

Table 4 shows the Pearson correlations and the partial coefficients between the two WCDS subscales (WCDS-S and WCDS-E), BDI-II and SASS. The correlation coefficients all proved significant. They indicated that the BDI-II was moderately correlated with the WCDS-S $(r=0.57$, $p<.001)$, WCDS-E $(r=0.48, p<.001)$, and SASS $(r=-0.57$, $p<.001$ ), and that the SASS was weakly correlated with the WCDS-S $(r=-0.39, p<.001)$ and WCDS-E $(r=-0.28, p<.001)$. Regarding the partial coefficients, the BDI-II was moderately correlated with the SASS ( $r=-0.47, p<.001$ ), and weakly correlated with the WCDS-S $(r=0.26, p<.001)$ and WCDS-E $(r=0.13, p<.001)$. The WCDS-S was weakly correlated with the SASS $(r=-0.12, p<.001)$, whereas the partial coefficient between the WCDS-E and SASS was not significant $(r=0.07, p=.18)$.

\subsubsection{Covariance structure analysis}

A covariance structure analysis was performed to determine the causal relationships between the WCDS-S, WCDS-E, BDI-II, and SASS. We removed pathways (from among those shown in Table 4) when the two variables exhibited a nonsignificant correlation coefficient, leaving a model fit for the analysis, as shown in Fig. $2\left(\chi^{2}=1.797 ; d f=1, n s\right.$; GFI $=0.998$; AGFI $=0.979$; CFI $=0.999$; RMSEA $=\leq 0.05$; with 90\% CIs: 0.144). The model in Fig. 2 satisfied the goodness-of-fit criteria (a good fit: GFI $>0.95$, AGFI $>0.90$, CFI $>0.97$, and
RMSEA < 0.05), and the results indicated that both of the cognitive distortion subscales could indirectly affect social adaptation via depressive symptoms, and that the WCDS-S directly affects social adaptation.

The WCDS-S $(\beta=0.466, p<.001)$ and WCDS-E $(\beta=0.135$, $p<.05$ ) predicted the BDI-II, together explaining $32.9 \%$ of its variance. The WCDS-S influenced the BDI-IIto a greater extent than the WCDS-E, as indicated by the larger $\beta$ value. The SASS was predicted directly by the BDI-II $(\beta=-0.516, p<.001)$ and WCDS-S $(\beta=-0.096$, $p<.001)$. The indirect effect of the WCDS-S on the SASS was $\beta=-0.240$ and the indirect effect of the WCDS-E on this outcome was $\beta=-0.070$. The WCDS-S, WCDS-E, and BDI-IItogether explained $33.1 \%$ of the variance of the SASS. The WCDS-S affected social adaptation indirectly more than it did directly.

The model (Fig. 2) that differentiated between the subscales exhibited a greater goodness-of-fit than the model that only analyzed the total scores of the WCDS (Fig. 1). Accordingly, we deemed the model involving the two subscales of the WCDS to be more valid.

\section{Discussion}

This study examined the relationship between cognitive distortion, depressive symptoms, and social adaptation. The covariance structure analysis indicated that the best model of this relationship was the one shown in Fig. 2, which included self-contained (WCDS-S) and environment-dependent (WCDS-E) cognitive distortions.

The study revealed that both of the cognitive distortion subscales affected social adaptation indirectly via depressive symptoms. Moreover, the WCDS-S directly affected social adaptation. Cognitive distortion and depressive symptoms explained approximately $33 \%$ of the variance in social adaptation. It was further revealed that the WCDS-S exerted a greater effect on depressive symptoms than the WCDS-E. Social adaptation was affected, in descending order of magnitude, indirectly by the WCDS-S, directly by the WCDS-S, and indirectly by the WCDS-E.

The modeled variables of social adaptation suggest that improving depressive symptoms is essential for achieving more socially adaptive outcomes. Therefore, the alleviation of symptoms is important. The results of the study support previous research that has found that reducing the severity of depressive symptoms explains more if not all of concurrent improvements in psychosocial functioning (Vittengl et al., 2004; Renner et al., 2014). However, given that cognitive distortion affected social adaptation indirectly via depressive symptoms, and that the WCDS-S directly affected social adaptation, cognitive assessment and intervention may also be effective for improving social adaptation. Scott et al. (2000) reported that the pharmacotherapy plus CT group showed significantly greater improvements in depressive symptoms and social function in the acute phase compared to the pharmacotherapy alone group. In addition, Lester et al. (2011) studied the effects of cognitive restructuring on individuals who interpreted uncertain circumstances as threatening. They reported that the intervention led to a more positive future outlook and increased motivation to engage in adaptive behavior. These results may be due to the fact that cognitive distortion not only affects the symptoms but also directly affects social adaptation, as shown in Fig. 2. In other words, this direct influence has an impact on a person's perception of their interaction with themselves and others and their social roles. It may also raise or lower an individual's readiness for adaptive behavior (e.g., motivation, interest, etc.). Therefore, cognitive interventions such as CT may have a facilitating role in improving social adaptation. Considering these things, it may be better to use not only pharmacotherapy but also CT to improve social adaptation.

We also found that the WCDS-S and WCDS-E separately affected depressive symptoms and social adaptation. This finding implies that the WCDS-S has a greater effect on depressive symptoms and social adaptation than the WCDS-E. In 1967, Beck defined six systematic 
errors in thinking (Beck, 1967). Since then, these errors have been renamed and extended by successive researchers (Carrie and Ditomasso, 2005). These categories have provided a clinically useful framework, helping the therapist and patient alike to understand the cognitive structure in clinical therapy. However, a clear categorization of cognitive distortion has proven difficult; attempts to develop tools for measuring cognitive distortion have so far failed to yield clear-cut categories, and some of the categories overlap (Covin et al., 2011). Although the literature has failed to clearly categorize the various cognitive distortions, it has demonstrated the feasibility of distinguishing between depressed and non-depressed cases (i.e., between those with cognitive distortion and those without). The literature also discusses the clinical effectiveness of quantifying the degree of cognitive distortion (Özdel et al., 2014). In this study, we demonstrated that two subcomponents of cognitive distortion exert separate effects. We also demonstrated that a model describing how each WCDS subscale affects depressive symptoms and social adaptation offers a better fit than a model describing how the total WCDS score affects these items. These findings imply that professionals, when attempting to treat depression and improve social adaptation, should not only quantitatively consider the degree of cognitive distortion in the patient but also qualitatively consider the type of cognitive distortion that the patient exhibits.

One such type is the WCDS-S, in which the person experiences negative thoughts as a result of judging matters chiefly by his or her own standards, as opposed to considering mainly the social environment. Numerous studies have highlighted a common cognitive bias: individuals often ignore information that contradicts their self-concept in order to maintain their self-consistency (e.g., Tesser and Campbell, 1984; Greenwald and Banaji, 1989; Swann et al., 1992; Brown, 1993). Evidence also suggests that individuals with depression tend to focus on stimuli that carry negative meaning, struggle to avert their attention from such stimuli, and lack the cognitive control to process negative information (e.g., Heatherton, 2011; Foland-Ross and Gotlib, 2012). Individuals who tend to interpret matters using their own criteria (as is the case in the WCDS-S) may fail to think objectively and flexibly, and thus perpetuate dysfunctional schemas of depression, resulting in depressive symptoms and impaired social adaptation. Therefore, when treating depression with a view to improving social adaptation, instead of only providing CT, professionals should measure the patient's cognitive distortion in order to identify the degree to which the patient suffers from WCDS-S. They should then formulate a treatment plan accordingly and assess its effectiveness.

Furthermore, in recent years, cognitive impairments such as attention deficit and disability of executive functions that highlight a certain rigidity in shifting the focus have been pointed out (Marazziti et al., 2010), and information processing distortion such as cognitive distortion may be also considered as a symptom of cognitive impairment (Gonda et al., 2015). Moreover, in treatment of well-known types of cognitive distortion in depression such as negative schemata, patients are taught by tasks involving working memory, inhibitory processes, and problem solving to be able to exert cognitive control over their topdown negative biases. Impaired neurocognitive function thus is suggested to contribute to the development of negative schemata (Roiser et al., 2012; Gonda et al., 2015). Since we did not directly investigate the relationship between cognitive distortion assessed with WCDS and neurocognitive function, it is unclear whether these two factors may have a causal relationship. However, considering that WCDS measures how events in the work environment are interpreted by people, that is, what attitude is more likely to be adopted to judge the events, impaired neurocognitive function such as cognitive rigidity due to dysfunctional executive function might have a role in the formation of cognitive distortion assessed with WCDS. Further direct examination of the relationships between these two factors is required to elucidate the nature of their relationships.

\subsection{Limitations}

This study has some limitations. First, we conducted the study on the premise that healthy cases are on a continuum with clinical cases. As such, one must be cautious about applying the results of healthy cases to clinical cases. Second, there have been few studies of the causal relationship between cognitive distortion and social adaptation; as such, further studies of this matter are required. Third, the participants in this study were engaged in the manufacturing industry, and they had a high rate of depressive symptoms and a low rate of social adaptation. Therefore, there is a possibility that membership bias exists. Future research should focus on a clinical sample and examine whether the causal relationships observed in healthy groups also exist in patients with depressive disorder, and whether interventions in cognitive distortion improve their social adaptation, while also considering the impact of pharmacotherapy. It would also be desirable to conduct a prospective study with a view to identifying an empirical therapy that addresses depression and impaired social adaptation.

\section{Funding}

This work was supported by a grant from the Mental Health Okamoto Memorial Foundation[grant number 47].

\section{CRediT authorship contribution statement}

Maki Ota: Conceptualization, Writing - original draft, Formal analysis, Writing - review \& editing. Shinya Takeda: Formal analysis, Writing - original draft, Writing - review \& editing. Shenghong Pu: Formal analysis, Writing - original draft, Writing - review \& editing. Hiroshi Matsumura: Writing - original draft, Writing - review \& editing. Takayuki Araki: Writing - original draft, Writing - review \& editing. Naoko Hosoda: Writing - original draft, Writing - review \& editing. Yoko Yamamoto: Writing - original draft, Writing - review \& editing. Aya Sakakihara: Methodology, Writing - review \& editing. Koichi Kaneko: Writing - original draft, Writing - review \& editing.

\section{Declarations of competing interest}

None.

\section{Acknowledgments}

We thank the employees who completed the survey. We would like to thank Editage (www.editage.jp) for English language editing.

\section{Supplementary materials}

Supplementary material associated with this article can be found, in the online version, at doi:10.1016/j.jad.2020.01.094.

\section{References}

Angst, J., 1997. Fortnightly review: a regular review of the long-term follow up of depression. BMJ 315, 1143-1146. https://doi.org/10.1136/bmj.315.7116.1143.

Beck, A.T., 1967. Depression: Clinical, Experimental and Theoretical Aspects. Harper and Row, New York.

Beck, A.T., 1976. Cognitive Therapy and the Emotional Disorders. Penguin, Harmondsworth, UK.

Beck, A.T., Shaw, B.W., Rush, A.J., Emery, G., 1979. Cognitive Therapy of Depression. Guilford Press, New York.

Beck, A.T., Steer, R.A., Brown, G.K., 1996. Manual For the Beck Depression Inventory-II. Psychological Corporation, San Antonio, TX.

Bosc, M., 2000. Assessment of social functioning in depression. Compr. Psychiat. 41, 63-69. https://doi.org/10.1016/S0010-440X(00)90133-0.

Bosc, M., Dubini, A., Polina, V., 1997. Development and validation of a social functioning scale, the Social Adaptation Self-Evaluation Scale. Eur. Neuropsychopharmacol. 7, 
57-70. https://doi.org/10.1016/S0924-977X(97)00420-3.

Briley, M., Moret, C., 2010. Improvement of social adaptation in depression with serotonin and norepinephrine reuptake inhibitors. Neuropsychiatr. Dis. Treat. 6, 647-655. https://doi.org/10.2147/NDT.S13171.

Brown, J.D., 1993. Motivational conflict and the self: the double-bind of low self-esteem. In: Baumeister, R.F. (Ed.), Self-Esteem: The Puzzle of Low Self-Regard. Plenum, New York, pp. 117-130.

Carrie, L.Y., Ditomasso, R.A., 2005. Cognitive distortions. In: Freeman, A., Felgoise, S.H., Nezu, C.M., Nezu, A.M., Reinecke, M.A. (Eds.), Encyclopedia of Cognitive Behavior Therapy. Springer, New York, pp. 117-122.

Covin, R., Dozois, D.J.A., Ogniewicz, A., Seeds, P.M., 2011. Measuring cognitive errors: initial development of the Cognitive Distortions Scale (CDS). Int. J. Cogn. Ther. 4, 297-322. http://dx.doi.org/10.1521/ijct.2011.4.3.297.

Cox, B.J., Enns, M.W., Borger, S.C., Parker, J.D.A., 1999. The nature of the depressive experience in analogue and clinically depressed samples. Behav. Res. Ther. 37, 15-24. https://doi.org/10.1016/S0005-7967(98)00107-7.

Coyne, J.C., 1976. Depression and the response of others. J. Abnorm. Psychol. 85, 186-193. http://doi.org/10.1037/0021-843X.85.2.186.

Ervasti, J., Vahtera, J., Pentti, J., Oksanen, T., Ahola, K., Kivimäki, M., Virtanen, M., 2013. Depression-related work disability: socioeconomic inequalities in onset, duration and recurrence. PLoS ONE 8, 11. https://doi.org/10.1371/journal.pone. 0079855.

Flett, G.L., Vredenburg, K., Krames, L., 1997. The continuity of depression in clinical and nonclinical samples. Psychol. Bull. 121, 395-416. https://doi.org/10.1037//00332909.121.3.395.

Foland-Ross, L.C., Gotlib, I.H., 2012. Cognitive and neural aspects of information processing in major depressive disorder: an integrative perspective. Front. Psychol. 3 (489), 1-17. https://doi.org/10.3389/fpsyg.2012.00489.

Gonda, X., Pompili, M., Serafini, G., Carvalho, A.F., Rihmer, Z., Dome, P., 2015. The role of cognitive dysfunction in the symptoms and remission from depression. Ann. Gen. Psychiatr 27, 14-27. http://doi.org/10.1186/s12991-015-0068-9.

Goto, M., Ueda, N., Yoshimura, R., Kakihara, S., Kaji, K., Yamada, Y., Shinkai, K., Nakashima, M., Iwata, N., Higuchi, T., Nakamura, J., 2005. Reliability and validity of the Japanese version of the Social Adaptation Self-Evaluation Scale (SASS). Clin. Psychiatry. 47, 483-489. in Japanese. https://doi.org/10.11477/mf.1405100063.

Greenwald, A.G., Banaji, M.R., 1989. The self as a memory system: powerful, but ordinary. J. Pers. Soc. Psychol. 57, 41-54. https://doi.org/10.1037/0022-3514.57. 1.41 .

Healy, D., 2000. Reboxetine: its effects as measured by the Social Adaptation SelfEvaluation Scale. Acta Psychiatr. Scand. 101, 45-51.

Heatherton, T.F., 2011. Neuroscience of self and self-regulation. Annu. Rev. Psychol. 62, 363-390. https://doi.org/10.1146/annurev.psych.121208.131616.

Hedlund, S., Rude, S.S., 1995. Evidence of latent depressive schemas in formerly depressed individuals. J. Abnorm. Psychol. 104, 517-525. https://doi.org/10.1037// 0021-843X.104.3.517.

Ishikawa, H., Kawakami, N., Kessler, R.C., Collaborators, W.M.H.J.S., 2016. Lifetime and 12-month prevalence, severity and unmet need for treatment of common mental disorders in Japan: results from the final dataset of World Mental Health Japan Survey. Epidemiol. Psych. Sci. 25, 217-229. https://doi.org/10.1017/ S2045796015000566.

Keller, M., 2001. Role of serotonin and noradrenaline in social dysfunction: a review of data on reboxetine and the Social Adaptation Self-Evaluation Scale (SASS). Gen. Hosp. Psychiatry. 23, 15-19. https://doi.org/10.1016/S0163-8343(00)00115-8.

Kojima, M., Furukawa, T.A., Takahashi, H., Kawai, M., Nagaya, T., Tokudome, S., 2002. Cross-cultural validation of the Beck depression inventory-II in Japan. Psychiatry Res. 110, 291-299. https://doi.org/10.1016/S0165-1781(02)00106-3.

Kupferberg, A., Bicks, L., Hasler, G., 2016. Social functioning in major depressive disorder. Neurosci. Biobehav. Rev. 69, 313-332. https://doi.org/10.1016/j.neubiorev. 2016.07.002.

Lester, K.J., Mathews, A., Davison, P.S., Burgess, J.L., Yiend, J., 2011. Modifying cognitive errors promotes cognitive well-being: a new approach to bias modification. J.
Behav. Ther. Exp. Psychiatry. 42, 298-308. https://doi.org/10.1016/j.jbtep.2011.01. 001.

Lewinsohn, P.M., Youngren, M.A., Grosscup, S.J., 1979. Reinforcement and depression. In: Depue, R.A. (Ed.), The Psychobiology of Depressive Disorders: Implications for the Effects of Stress. Academic Press, New York, pp. 291-315.

Libet, J.M., Lewinsohn, P.M., 1973. Concepts of social skill with special reference to the behavior of depressed persons. J. Consult. Clin. Psychol. 40, 304-312.

Marazziti, D., Consoli, G., Picchetti, M., Carlini, M., Faravelli, L., 2010. Cognitive impairment in major depression. Eur. J. Pharmacol. 626, 83-86. https://doi.org/10. 1016/j.ejphar.2009.08.046.

Marco, J.H., Alonso, S., Andani, J., 2018. Early intervention with cognitive behavioral therapy reduces sick leave duration in people with adjustment, anxiety and depressive disorders. J. Ment. Health. 27, 1-9. https://doi.org/10.1080/09638237.2018. 1521937.

Matsunaga, M., Okamoto, Y., Suzuki, S., Kinoshita, A., Yoshimura, S., Yoshino, A., Kunisato, Y., Yamawaki, S., 2010. Psychosocial functioning in patients with treatment-resistant depression after group cognitive behavioral therapy. BMC Psychiatry 10, 22. https://doi.org/10.1186/1471-244X-10-22.

Organisation for Economic Co-operation and Development, 2012. Sick on the Job? Myths and Realities About Mental Health and Work. OECD Publishing, Paris. https://doi. org/10.1787/22257985.

Ota, M., Takeda, S., Hamada, M., Inoue, M., 2014. Evaluation of the Worker's Cognitive Distortion Scale (WCDS). Jpn. J. Cogn. Ther. 7, 76-83 in Japanese.

Özdel, K., Taymur, I., Guriz, S.O., Tulaci, R.G., Kuru, E., Turkcapar, M.H., 2014. Measuring cognitive errors using the Cognitive Distortions Scale (CDS): psychometric properties in clinical and non-clinical samples. PLoS ONE 9, e105956. https://doi. org/10.1371/journal.pone.0105956.

Rehm, L.P., 1977. A self-control model of depression. Behav. Ther. 8, 787-804. https:// doi.org/10.1016/S0005-7894(77)80150-0.

Renner, F., Cuijpers, P., Huibers, M.J.H., 2014. The effect of psychotherapy for depression on improvements in social functioning: a meta-analysis. Psychol. Med. 44, 2913-2926. http://doi.org/10.1017/S0033291713003152.

Roiser, J., Elliott, R., Sahakian, B., 2012. Cognitive mechanisms of treatment in depression. Neuropsychopharmacol 37, 117-136. http://doi.org/10.1038/npp.2011.183.

Schermelleh-Engel, K., Moosbrugger, H., Müller, H., 2003. Evaluating the fit of structural equation models: tests of significance and descriptive goodness-of-fit measures. Meth. Psychol. Res. 8, 23-74.

Scott, J., Teasdale, J.D., Paykel, E.S., Johnson, A.L., 2000. Effects of cognitive therapy on psychological symptoms and social functioning in residual depression. Br. J. Psychiatry 177, 440-446. https://doi.org/10.1192/bjp.177.5.440.

Seligman, M.E.P., 1981. A learned helplessness point of view. In: Rehm, L.P. (Ed.), Behavior Therapy for Depression: Present Status and Future Directions. Academic Press, New York, pp. 123-142.

Sheehana, D.V., Nakagome, K., Asami, Y., Pappadopulos, E.A., Boucher, M., 2017. Restoring function in major depressive disorder: a systematic review. J. Affect. Disorder. 215, 299-313. https://doi.org/10.1016/j.jad.2017.02.029.

Swann Jr., W.B., Stein-Seroussi, A., Giesler, R.B., 1992. Why people self-verify. J. Pers. Soc. Psychol. 62, 392-401.

Tesser, A., Campbell, J., 1984. Self-definition and self-evaluation maintenance. In: Suls, J., Greenwald, A. (Eds.), Psychological Perspectives on the Self. Erlbaum. Hillsdale, NJ, pp. 1-32.

Ueda, N., Suda, A., Nakagawa, M., Nakano, H., Umene-Nakano, W., Ikenouchi-Sugita, A., Hori, H., Yoshimura, R., Nakamura, J., 2011. Reliability, validity and clinical utility of a Japanese version of the Social Adaptation Self-Evaluation Scale as calibrated using the Beck depression inventory. Psychiatry Clin. Neurosci. 65, 624-629. https:// doi.org/10.1111/j.1440-1819.2011.02274.x.

Vittengle, J.R., Clark, L.A., Jarrett, R.B., 2004. Improvement in social-interpersonal functioning after cognitive therapy for recurrent depression. Psychol. Med. 34, 643-658. https://doi.org/10.1017/S0033291703001478.

Weishaar, M., 1993. Aaron T. Beck. Sage, London. 